PROCEEDINGS OF THE

AMERICAN MATHEMATICAL SOCIETY

Volume 126, Number 7, July 1998, Pages 2053-2060

S $0002-9939(98) 04251-8$

\title{
LAPLACIANS AND SOBOLEV GRADIENTS
}

\author{
J. W. NEUBERGER
}

(Communicated by Christopher D. Sogge)

\begin{abstract}
We describe a class of operators which fit the description of laplacians and which may be used to unify the construction of various Sobolev gradients.
\end{abstract}

\section{INTRODUCTION}

In [9] Weyl discusses the problem of determining when a vector field is the gradient of some function. He introduces a method of orthogonal projections to solve this problem for all square integrable (but not necessarily differentiable) vector fields. In the construction of Sobolev gradients for problems in nonlinear partial differential equations [6] a family of problems of a somewhat similar nature arises. Using an idea of Beurling and Deny $[2],[3]$ we give a generalization of the LaxMilgram Theorem [5] which unifies a wide class of Sobolev gradient constructions.

\section{A constrained LaX-Milgram theorem}

Suppose that each of $H, H^{\prime}, H_{0}^{\prime}$ is a Hilbert space (all Hilbert spaces in this note are real) so that

$$
\begin{gathered}
H_{0}^{\prime} \subset H^{\prime} \subset H, \\
\|x\|_{H_{0}^{\prime}}=\|x\|_{H^{\prime}}, x \in H_{0}^{\prime},
\end{gathered}
$$

and

$$
\|x\|_{H} \leq\|x\|_{H^{\prime}}, x \in H^{\prime} .
$$

Suppose also that the points of $H_{0}^{\prime}, H^{\prime}$ are dense in $H$.

For an example, take

$$
H=L_{2}([0,1]), H^{\prime}=H^{1,2}([0,1])
$$

and

$$
H_{0}^{\prime}=\left\{f \in H^{\prime}: f(0)=0=f(1)\right\}
$$

([1] is a general reference for Sobolev spaces).

Following Beurling-Deny [2],[3], for the pair $\left(H, H^{\prime}\right)$ there is an associated transformation called the laplacian for $\left(H, H^{\prime}\right)$. It is described as follows. Pick $y \in H$ and denote by $f$ the functional on $H$ corresponding to $y$ :

$$
f(x)=\langle x, y\rangle_{H}, x \in H .
$$

Received by the editors March 15, 1996 and, in revised form, December 18, 1996.

1991 Mathematics Subject Classification. Primary 35A15; Secondary 47F05.

Key words and phrases. Laplacian, Sobolev gradient.

(C) 1998 American Mathematical Society 
Denote by $k$ the restriction of $f$ to $H^{\prime}$. Then

$$
|k(x)|=\left|\langle x, y\rangle_{H}\right| \leq\|x\|_{H}\|y\|_{H} \leq\|x\|_{H^{\prime}}\|y\|_{H}, x \in H^{\prime} .
$$

Hence $k$ is a continuous linear functional on $H^{\prime}$ and so there is a unique member $z$ of $H^{\prime}$ such that

$$
k(x)=\langle x, z\rangle_{H^{\prime}}, x \in H^{\prime} .
$$

Define $M: H \rightarrow H^{\prime}$ by $M y=z$ where $y, z$ are as above. We will see that $M^{-1}$ exists; it will be called the laplacian for the pair $\left(H, H^{\prime}\right)$. For the same process as above but with $H^{\prime}$ replaced by $H_{0}^{\prime}$ we denote the corresponding transformation by $M_{0}$.

Theorem 1. The following hold for $M$ as defined above: (1) $R(M)$, the range of $M$, is dense in $H$, (2) $|M|_{L\left(H, H^{\prime}\right)} \leq 1$, (3) $M^{-1}$ exists, (4) $M$, considered as a transformation from $H \rightarrow H$, is symmetric.

Proof. Suppose first that there is a nonzero $z \in H^{\prime}$ so that $\langle z, M x\rangle_{H^{\prime}}=0, x \in H$. Then $0=\langle z, M z\rangle_{H^{\prime}}=\langle z, z\rangle_{H}$ and so $z=0$, a contradiction. Thus $c l_{H^{\prime}} R(M)=H^{\prime}$. But then $H^{\prime}=c l_{H^{\prime}}(R(M)) \subset c l_{H}(R(M))$. Hence $H=c l_{H}\left(H^{\prime}\right)=c l_{H}(R(M))$ and (1) is demonstrated.

To show that (2) holds, suppose that $x \in H$. Then

$$
\begin{gathered}
\|M x\|_{H^{\prime}}=\sup _{z \in H^{\prime}, z \neq 0}\langle z, M x\rangle_{H^{\prime}} /\|z\|_{H^{\prime}} \\
=\sup _{z \in H^{\prime}, z \neq 0}\langle z, x\rangle_{H} /\|z\|_{H^{\prime}} \leq \sup _{z \in H, z \neq 0}\langle z, x\rangle_{H} /\|z\|_{H}=\|x\|_{H},
\end{gathered}
$$

and so $|M|_{L\left(H, H^{\prime}\right)} \leq 1$.

To show that (3) holds, suppose that $x \in H$ and $M x=0$. Then

$$
0=\langle z, M x\rangle_{H^{\prime}}=\langle z, x\rangle_{H}, z \in H^{\prime} .
$$

But this implies that $z=0$ since the points of $H^{\prime}$ are dense in $H$.

To see that (4) holds observe that if $x, z \in H$, then

$$
\langle z, M x\rangle_{H}=\langle M z, M x\rangle_{H^{\prime}}=\langle M z, x\rangle_{H} .
$$

Denote by $P_{0}$ the orthogonal projection of $H^{\prime}$ onto $H_{0}^{\prime}$ and denote the complementary projection $I-P_{0}$ by $Q_{0}$. The following is a slight extension of the Lax-Milgram [5] theorem.

Theorem 2. Suppose $g \in H, w \in H^{\prime}$ and $\beta: H^{\prime} \rightarrow R$ is defined by

$$
\beta(u)=(1 / 2)\|u\|_{H^{\prime}}^{2}-\langle u, g\rangle_{H}, u \in H^{\prime} .
$$

Then the minimum of $\beta(u)$ subject to the condition $Q_{0} u=Q_{0} w$ is achieved by

$$
u=Q_{0} w+M_{0} g .
$$

The condition $Q_{0} u=Q_{0} w$ may be regarded as a generalized boundary condition; it is equivalent to asking that $u-w \in H_{0}^{\prime}$. With $H, H_{0}^{\prime}, H^{\prime}$ as in the example, $Q_{0} u=Q_{0} w$ asserts that $u(1)=w(1), u(0)=w(0)$. More unusual conditions might be

$$
u(1 / 2)=w(1 / 2), u(3 / 4)-u(1 / 4)=w(3 / 4)-w(1 / 4) .
$$

This specification is done by defining

$$
H_{0}^{\prime}=\left\{f \in H^{1,2}([0,1]): f(1 / 2)=0, f(3 / 4)=f(1 / 4)\right\} .
$$


Such boundary conditions are not easily dealt with by using integration by parts, but such facts have essentially no impact in the present theory.

When $H^{\prime}=H^{1,2}(\Omega), \Omega \subset R^{2}$, the kinds of boundary conditions which fit the present development include many of uncharted character, some of which just might be of physical significance.

Proof. Define $q=Q_{0} w$ and define $\gamma: H_{0}^{\prime} \rightarrow R$ by

$$
\gamma(y)=\beta(y+q), y \in H_{0}^{\prime} .
$$

Note that

$$
\gamma^{\prime}(y) k=\beta^{\prime}(y+q) k=\langle y+q, k\rangle_{H^{\prime}}-\langle k, g\rangle_{H}, k \in H_{0}^{\prime} .
$$

Note also that since

$$
\gamma^{\prime \prime}(y)(k, k)=\|k\|_{H}^{2}, k, y \in H_{0}^{\prime},
$$

it follows that $\gamma$ is (strictly) convex. Now

$$
\begin{aligned}
\beta(u)=(1 / 2)\|u\|_{H^{\prime}}^{2}-\langle u, g\rangle_{H} \geq(1 / 2)\|u\|_{H^{\prime}}^{2}-\|u\|_{H}\|g\|_{H} \\
\geq(1 / 2)\|u\|_{H^{\prime}}^{2}-\|u\|_{H^{\prime}}\|g\|_{H}=\|u\|_{H^{\prime}}\left(\|u\|_{H^{\prime}} / 2-\|g\|_{H}\right), u \in H^{\prime},
\end{aligned}
$$

so $\beta$ and hence $\gamma$ are bounded from below.

Since $\gamma$ is convex and bounded from below it has an absolute minimum if and only if it has a critical point. Moreover, such a critical point would be the unique point at which $\beta$ attains its minimum. Observe that

$$
\gamma^{\prime}(y) k=\langle y+q, k\rangle_{H^{\prime}}-\langle k, g\rangle_{H}=\langle y, k\rangle_{H^{\prime}}-\langle k, g\rangle_{H}
$$

since $\langle q, k\rangle_{H^{\prime}}=0, k \in H_{0}^{\prime}$. Now

$$
\langle k, g\rangle_{H}=\langle y, k\rangle_{H^{\prime}}, k \in H_{0}^{\prime},
$$

if and only if $y=M_{0} g$. Choosing $y$ in this way thus yields a critical point of $\gamma$. Consequently $u=y+q$ is the point of $H^{\prime}$ at which $\beta$ attains its minimum. Therefore

$$
u=Q_{0} w+M_{0} g
$$

is the point at which $\beta$ attains its minimum and the theorem is proved.

\section{LAPLACIANS AND CLOSED LINEAR TRANSFORMATIONS}

We now turn to a somewhat more concrete case of the above - a case which is closer to the example.

Suppose that each of $H$ and $K$ is a Hilbert space and $T$ is a closed and densely defined linear transformation on $H$ to $K$. Let $H^{\prime}$ be the Hilbert space whose points are those of $D(T)$ where

$$
\|x\|_{H^{\prime}}=\left\|\left(\begin{array}{c}
x \\
T x
\end{array}\right)\right\|_{H \times K}=\left(\|x\|_{H}^{2}+\|T x\|_{K}^{2}\right)^{1 / 2}, x \in D(T)
$$

(the graph norm of $x$ relative to $T$ ). Suppose that the linear transformation $T_{0}$ is a closed, densely defined restriction of $T$ (see [4],[7] for a discussion of closed unbounded linear operators from one Hilbert space to another). Denote by $H_{0}^{\prime}$ the Hilbert space whose points are those of $D\left(T_{0}\right)$ where

$$
\|x\|_{H_{0}^{\prime}}=\left\|\left(\begin{array}{c}
x \\
T_{0} x
\end{array}\right)\right\|_{H \times K}, x \in D\left(T_{0}\right) .
$$

Then $H, H^{\prime}, H_{0}^{\prime}$ fit the hypothesis of Theorem 2.

We remind the reader of the definition of $T^{t}$. The domain of $T^{t}$ is

$$
\left\{y \in H: x \rightarrow\langle T x, y\rangle_{K} \text { is continuous }\right\} .
$$


For $y \in D\left(T^{t}\right), T^{t} y$ is the element of $H$ such that

$$
\langle T x, y\rangle_{K}=\left\langle x, T^{t} y\right\rangle_{H}, x \in D(T) .
$$

The definition of adjoint applies just as well when $T$ is replaced by $T_{0}$.

One can choose $T$ to be a differential operator in such a way that the resulting space $H^{\prime}$ is one of the classical Sobolev spaces which is also a Hilbert space. Then the restriction $T_{0}$ of $T$ can be chosen so that $H_{0}^{\prime}$ is a subspace of $H^{\prime}$ consisting of those members of $H^{\prime}$ which satisfy zero boundary conditions in some sense (much more variety than this can be accommodated). In the example, $T$ is the derivative operator whose domain consists of the elements of $H^{1,2}([0,1])$. In other cases $T$ might be a gradient operator.

Theorem 3. Suppose $g \in H, w \in H^{\prime}$ and $\beta$ satisfies (2.1). Then the element of $H^{\prime}$ satisfying the conclusion to Theorem 2 is the unique solution $u$ to

$$
\left(I+T_{0}{ }^{t} T\right) u=g, Q_{0} u=Q_{0} w
$$

where $Q_{0}$ is as in Theorem 2 in its relationship with $H^{\prime}, H_{0}^{\prime}$.

In the example, $\left(I+T_{0}{ }^{t} T\right)$ is the differential operator so that

$$
\left(I+T_{0}^{t} T\right) u=u-u^{\prime \prime}
$$

for all $u$ in its domain (without any boundary conditions on its domain - that is, it is the maximal operator associated with its expression).

Proof. From Theorem 2, the minimum $u$ of $\beta$, subject to $Q_{0} u=Q_{0} w$, may be written

$$
u=Q_{0} w+M_{0} g .
$$

It is clear that for $u$ defined in this way, $Q_{0} u=Q_{0} w$ since $R\left(M_{0}\right) \subset R\left(P_{0}\right)$ and $Q_{0}=I-P_{0}$. It remains to show that

$$
\left(I+T_{0}^{t} T\right) u=g .
$$

We first show that

$$
\left(I+T_{0}^{t} T\right) Q_{0} w=0
$$

To this end, first note that

$$
\left\langle Q_{0} w, x\right\rangle_{H_{0}^{\prime}}=0, x \in H_{0}^{\prime},
$$

since $x=P_{0} x, x \in H_{0}^{\prime}$. This may be rewritten

$$
\left\langle\left(\begin{array}{c}
Q_{0} w \\
T Q_{0} w
\end{array}\right),\left(\begin{array}{c}
x \\
T_{0} x
\end{array}\right)\right\rangle_{H \times K}=0, x \in D\left(T_{0}\right) .
$$

But this is equivalent to

$$
\left\langle T_{0} x, T Q_{0} w\right\rangle_{K}=\left\langle x,-Q_{0} w\right\rangle_{H}, x \in D\left(T_{0}\right),
$$

and hence

$$
T Q_{0} w \in D\left(T_{0}^{t}\right)
$$

and

$$
T_{0}^{t} T Q_{0} w=-Q_{0} w
$$

that is,

Next we show that

$$
\left(I+T_{0}^{t} T\right) Q_{0} w=0
$$

$$
\left(I+T_{0}^{t} T\right) M_{0} g=g
$$


To do this first note that $M_{0} g \in D\left(T_{0}\right)$ since $M_{0} g \in H_{0}^{\prime}$ and so $T M_{0} g=T_{0} M_{0} g$. Using the definition of $M_{0}$,

$$
\langle x, g\rangle_{H}=\left\langle x, M_{0} g\right\rangle_{H_{0}^{\prime}}
$$

and so

$$
\langle x, g\rangle_{H}=\left\langle x, M_{0} g\right\rangle_{H}+\left\langle T_{0} x, T_{0} M_{0} g\right\rangle_{K}
$$

that is,

$$
\left\langle T_{0} x, T_{0} M_{0} g\right\rangle_{K}=\left\langle x, g-M_{0} g\right\rangle_{H}, x \in D\left(T_{0}\right) .
$$

But this implies that

$$
T_{0} M_{0} g \in D\left(T_{0}^{t}\right)
$$

and

$$
T_{0}^{t} T_{0} M_{0} g=g-M_{0} g
$$

that is,

$$
\left(I+T_{0}^{t} T_{0}\right) M_{0} g=g
$$

and the argument is complete.

The expression

$$
\left(I+T_{0}^{t} T_{0}\right)
$$

is the inverse of $M_{0}$ and is called the laplacian associated with the pair $\left(H, H_{0}^{\prime}\right)$. Similarly the expression

$$
\left(I+T^{t} T\right)
$$

is the laplacian associated with the pair $\left(H, H^{\prime}\right)$. The expression

$$
\left(I+T_{0}^{t} T\right)
$$

plays the role of maximal operator associated with the triple $H, H^{\prime}, H_{0}^{\prime}$. Theorem 3 gives that $R\left(I+T_{0}^{t} T\right)=H$. One may observe that $N\left(I+T_{0}^{t} T\right)$ is the orthogonal complement of $H_{0}^{\prime}$ in $H^{\prime}$.

\section{Sobolev GRadients}

Transformations (3.3),(3.4),(3.5) are related to the problem of constructing some orthogonal projections on $H \times K$. We will see that such projections have a central role in the construction of Sobolev gradients for problems in differential equations.

Notation in this section is the same as the previous section. We consider first the problem of constructing the orthogonal projection of $H \times K$ onto $T$, i.e., onto $\left.\left\{\begin{array}{c}x \\ T x\end{array}\right): x \in D(T)\right\}$. We use a result of Von Neumann [8].

Theorem 4. If $T$ is a closed, densely defined linear transformation on $H$ to $K$ and $(f, g) \in H \times K$, then the nearest point of $T$ to $(\underset{g}{f})$ is the element

$$
\left(\left(I+T^{t} T\right)^{-1} f+T^{t}\left(I+T T^{t}\right)^{-1} g, T\left(I+T^{t} T\right)^{-1} f+\left(g-\left(I-T T^{t}\right)^{-1} g\right)\right) .
$$

Proof. The transformation (call it $P_{T}$ ) which takes $(f, g) \in H \times K$ into (4.1) is linear, symmetric, idempotent, fixed on elements of the form $(x, T x), x \in D(T)$, and has range equal to this set. This is enough to convict $P_{T}$ of being the required projection. 
This writer thanks Philip Walker for calling his attention to this result. Note that (4.1) contains both

$$
\left(I+T^{t} T\right)^{-1} \text { and }\left(I+T T^{t}\right)^{-1},
$$

the latter being the inverse of the laplacian developed on $K \times H$ for $T^{t}$.

Theorems 5 and 6 below deal with relationships between two different adjoints for $T$. One is $T^{t}$ with $T$ is considered as a closed densely defined linear transformation on $H$ to $K$ and the other is $T^{*}$ with $T$ considered as a member of $L\left(H^{\prime}, K\right)$ where $H^{\prime}$ is the Hilbert space whose points are those of $D(T)$ but with

$$
\|x\|_{H^{\prime}}=\left(\|x\|_{H}^{2}+\|T x\|_{K}^{2}\right)^{1 / 2}, x D(T) .
$$

The following gives a relationship between $T^{t}$ and $T^{*}$. We use the notations $T^{t}, T^{*}$ for adjoint here in order to be consistent with previous usage in work on Sobolev gradients, for example in [6]. We denote by $\pi$ the transformation from $H \times K$ to $H$ defined by

\section{Theorem 5 .}

$$
\pi\left(\begin{array}{l}
f \\
g
\end{array}\right)=f,\left(\begin{array}{l}
f \\
g
\end{array}\right) \in H \times K .
$$

$$
T^{*} y=\pi P_{T}\left(\begin{array}{l}
0 \\
y
\end{array}\right), y \in K .
$$

Proof. If $x \in D(T), y \in K$, then

$$
\langle T x, y\rangle_{K}=\left\langle\left(\begin{array}{c}
x \\
T x
\end{array}\right),\left(\begin{array}{l}
0 \\
y
\end{array}\right)\right\rangle_{H \times K}=\left\langle\left(\begin{array}{c}
x \\
T x
\end{array}\right), P_{T}\left(\begin{array}{l}
0 \\
y
\end{array}\right)\right\rangle_{H \times K}=\left\langle x, \pi P_{T}\left(\begin{array}{l}
0 \\
y
\end{array}\right)\right\rangle_{H^{\prime}}
$$

so that the conclusion holds.

For the case in which

$$
H=H^{1,2}([0,1]), K=L_{2}([0,1]), T f=f^{\prime}, f \in H,
$$

the reader may want to calculate an explicit expression for $T^{*}$. An answer is in [6].

Returning to our general setting, a type of transformation often used in constructing Sobolev gradients is $E$ such that

$$
D(E)=D(T), E x=\left(\begin{array}{c}
x \\
T x
\end{array}\right), x \in D(T) .
$$

\section{Theorem 6.}

$$
E^{*}\left(\begin{array}{l}
f \\
g
\end{array}\right)=\pi P_{T}\left(\begin{array}{l}
f \\
g
\end{array}\right),\left(\begin{array}{l}
f \\
g
\end{array}\right) \in H \times K .
$$

Proof. Suppose $x \in H=D(T),\left(\begin{array}{l}f \\ g\end{array}\right) \in H \times K$. Then

$$
\left\langle E x,\left(\begin{array}{c}
f \\
g
\end{array}\right)\right\rangle_{H \times K}=\left\langle\left(\begin{array}{c}
x \\
T x
\end{array}\right),\left(\begin{array}{l}
f \\
g
\end{array}\right)\right\rangle_{H \times K}=\left\langle\left(x, \pi P_{T}\left(\begin{array}{l}
f \\
g
\end{array}\right)\right\rangle_{H^{\prime}}\right.
$$

so that the conclusion follows.

By contrast we have that if $x \in D(E),\left(\begin{array}{l}f \\ g\end{array}\right) \in H \times K$, then

$$
\begin{gathered}
\left\langle E x,\left(\begin{array}{c}
f \\
g
\end{array}\right)\right\rangle_{H \times K}=\left\langle\left(\begin{array}{c}
x \\
T x
\end{array}\right),\left(\begin{array}{l}
f \\
g
\end{array}\right)\right\rangle_{H \times K} \\
=\langle x, f\rangle_{H}+\langle T x, g\rangle_{K}=\langle x, f\rangle_{H}+\langle x, T t\rangle_{H}=\left\langle x, f+T^{t} g\right\rangle_{H}
\end{gathered}
$$

provided $x \in D(T), g \in D\left(T^{t}\right)$. Thus for this case we have

$$
E^{t}\left(\begin{array}{l}
f \\
g
\end{array}\right)=f+T^{t} g \text {. }
$$

Following this pattern we denote by $P_{T_{0}}$ the orthogonal projection of $H \times K$ onto

$$
\left\{\left(\begin{array}{c}
x \\
T_{0} x
\end{array}\right): x \in D\left(T_{0}\right)\right\} .
$$

We now offer some indications of how such projections are related to Sobolev gradients for differential equations. Such gradients are useful in descent processes, 
both in function spaces and in finite dimensional emulations of function spaces [6] (for purposes of computation).

We first consider the problem of finding a critical point of $\phi$ :

$$
\phi(u)=\int_{\Omega} F(E u), u \in H^{\prime}=H^{1,2}(\Omega),
$$

where $F \in C^{2}\left(R^{m+1}, R\right), \Omega \subset R^{m}$. We fix boundary conditions by picking a closed subspace $H_{0}^{\prime}$ of $H^{\prime}$, picking $w \in H_{0}^{\prime}$ and requiring that a critical point $u$ satisfy $u-w \in H_{0}^{\prime}$. Take $T: H^{\prime} \rightarrow L_{2}(\Omega)^{m}$ defined by $T u=\nabla u, u \in H^{\prime}$. We pick $T_{0}$ to be the restriction of $T$ to $H_{0}^{\prime}$. We suppose that for $u \in H^{\prime}, \phi^{\prime}(u)$ is a continuous linear functional on $H^{\prime}$. With $E$ related to $T$ as above and for $u \in H^{\prime}, E u$ is a list consisting of $u$ and its partial derivatives. In the following result, if $V \in L_{2}(\Omega)^{m+1}$ then $\pi V$ will denote the first component of $V$.

Theorem 7. If $u$ is a critical point of $\phi$, then

$$
\pi P_{0}(\nabla F)(E u)=0 .
$$

Proof. Suppose $u \in H^{\prime}$ and $h \in H_{0}^{\prime}$. Then

$$
\begin{gathered}
\phi^{\prime}(u) h=\int_{\Omega} F^{\prime}(E u) E h=\langle E h,(\nabla F)(E h)\rangle_{L_{2}(\Omega)^{m+1}} . \\
=\left\langle E h, P_{T_{0}}(\nabla F)(E u)\right\rangle_{L_{2}(\Omega)^{m+1}}
\end{gathered}
$$

since $E h \in R\left(P_{T_{0}}\right)$. Thus

$$
\phi^{\prime}(u) h=\left\langle h, \pi P_{T_{0}}(\nabla F)(E u)\right\rangle_{H_{0}^{\prime}}, h \in H_{0}^{\prime}, u \in H^{\prime} .
$$

At a critical point $u$ of $\phi, \phi^{\prime}(u) h=0$ for all $h \in H_{0}^{\prime}$. Thus the conclusion holds.

Equation (4.2) is an appealing substitute for the usual Euler-Lagrange equation. For one thing, it requires only one derivative in order to make sense. For another, even if $u$ is not a critical point,

$$
(\nabla \phi(u))=\pi P_{0}(\nabla F)(E u),
$$

the gradient of $\phi$ at $u$, i.e, the element so that

$$
\phi^{\prime}(u) h=\langle h,(\nabla \phi)(u)\rangle_{H_{0}^{\prime}}, h \in H_{0}^{\prime} .
$$

The gradient function $\nabla \phi$ is continuous, even differentiable. It is suitable for use in continuous steepest descent:

$$
z(0)=x \in H^{\prime}, z^{\prime}(t)=-(\nabla \phi)(z(t)), t \geq 0
$$

(global existence on $[0, \infty)$ is assured, see [6]). Since

$$
(\nabla \phi)(z(t)) \in H_{0}^{\prime}, t \geq 0,
$$

and

it follows that

$$
z(t)-x=\int_{0}^{t}(\nabla \phi)(z), t \geq 0
$$

$$
P_{0}(z(t)-x)=z(t)-x, \text { i.e., } Q_{0}(z(t))=Q(x), t \geq 0,
$$

so that $z(t)$ and $x$ satisfy the same boundary conditions, $t \geq 0$. 
We now illustrate with an example how systems that do not arise from a conventional variational principle can also fit the present development. Suppose that $\Omega$ is a domain in $R^{2}$,

$$
H=L_{2}(\Omega), H^{\prime}=H^{1,2}(\Omega), H_{0}^{\prime} \subset H^{\prime} .
$$

Suppose also that

$$
G \in C^{2}\left(R^{3}, R\right), T u=\nabla u, E u=\left(\begin{array}{c}
u \\
T u
\end{array}\right), u \in H^{\prime}, w \in H^{\prime} .
$$

A first order partial differential equation is specified by the problem of finding $u \in H^{\prime}$ such that

$$
G(E u)=0
$$

with boundary conditions specified by requiring that $Q_{0} u=Q_{0} w, I-Q_{0}$ being the orthogonal projection of $H^{\prime}$ onto $H_{0}^{\prime}$. Form

$$
\phi: \phi(u)=\|G(E u)\|_{H}^{2} / 2, u \in H^{\prime} .
$$

Assume that $G$ is such that $\phi^{\prime}(u)$ is a continuous linear functional on $H^{\prime}$ if $u \in H^{\prime}$.

\section{Theorem 8.}

$$
(\nabla \phi)(u)=\pi P_{T_{0}} G^{\prime}(E u)^{t} G(E u) \in H_{0}^{\prime}, u \in H^{\prime} .
$$

Proof.

$$
\begin{gathered}
\phi^{\prime}(u) h=\left\langle G^{\prime}(E u) E h, G(E u)\right\rangle_{H},=\left\langle E h, G^{\prime}(E u)^{t} G(E u)\right\rangle_{L_{2}(\Omega)}^{3} \\
=\left\langle E h, P_{T_{0}} G^{\prime}(E u)^{t} G(E u)\right\rangle_{L_{2}(\Omega)^{3}}=\left\langle h, \pi P_{T_{0}} G^{\prime}(E u)^{t} G(E u)\right\rangle_{H_{0}^{\prime}},
\end{gathered}
$$

for all $h \in H_{0}^{\prime}$. Thus (4.3) holds.

This gradient is suitable for use with continuous descent in seeking $u$ such that $G(E u)=0$ :

$$
z(0)=x \in H^{\prime}, z^{\prime}(t)=-(\nabla \phi)(z(t)), t \geq 0 .
$$

For such $z, Q_{0} x=Q_{0}(z(t)), t \geq 0$ and so, if it exists

$$
u=\lim _{t \rightarrow \infty} z(t)
$$

satisfies $Q_{0} u=Q_{0} x$.

\section{REFERENCES}

1. R. A. Adams, Sobolev Spaces, Academic Press, 1978. MR 56:9247

2. A. Beurling and J. Deny, Dirichlet Spaces, Proceedings Nat. Acad. Sci. 45 (1959), 208-215. MR 21:5098

3. A. Beurling, Collected Works, Vol. 2, Editors: L. Carleson, P. Malliavin, J. Neuberger, J. Wermer, Birkhauser, 1989. MR 92k:01046b

4. H. Brezis, Analyse Fonctionnelle, Masson, 1993. MR 85a:46001

5. P. D. Lax, Parabolic Equations, Annls. Math. Stud. V, 167-190. MR 16:709b

6. J. W. Neuberger, Sobolev Gradients and Differential Equations, Lecture Notes in Mathematics \#1670, Springer, 1997.

7. F. Riesz and B. Sz.-Nagy, Functional Analysis, Ungar, 1955. MR 17:175i

8. J. VonNeumann, Functional Operators II, Annls. Math. Stud. 22, 1950. MR 11:599e

9. H. Weyl, The method of orthogonal projections in potential theory, Duke Math.J. 7 (1940), 411-444. MR 2:202a

Department of Mathematics, University of North Texas, Denton, Texas 76203

E-mail address: jwn@unt.edu 